\title{
Value of serology in predicting Pseudomonas aeruginosa infection in young children with cystic fibrosis
}

\author{
Tonia A Douglas, ${ }^{1,2}$ Siobhain Brennan, ${ }^{2}$ Luke Berry, ${ }^{2}$ Kaye Winfield, ${ }^{2}$ \\ Claire E Wainwright, ${ }^{3,4}$ Keith Grimwood, ${ }^{4,5}$ Stephen M Stick, ${ }^{1,2}$ Peter D Sly, ${ }^{1,2}$ \\ on behalf of the members of AREST CF and the ACFBAL Trial
}

\begin{abstract}
- Additional materials are published online only. To view these files please visit the journal online (http://thorax.bmj. com).

${ }^{1}$ Department of Respiratory Medicine, Princess Margaret Hospital for Children, Perth, Western Australia, Australia ${ }^{2}$ Telethon Institute for Child Health Research and Centre for Child Health Research, University of Western Australia, Perth, Western Australia, Australia

${ }^{3}$ Queensland Children's Respiratory Centre, Royal Children's Hospital, Queensland, Australia

${ }^{4}$ Oueensland Children's Medical Research Institute, The University of Queensland, Royal Children's Hospital, Brisbane, Queensland, Australia ${ }^{5}$ Queensland Paediatric Infectious Disease Laboratory, Royal Children's Hospital, Queensland, Australia
\end{abstract}

\section{Correspondence to} Dr Tonia Douglas, Department of Respiratory Medicine, Princess Margaret Hospital for Children, Roberts Road, Subiaco, Perth, WA 6008 Australia;

tonia.douglas@health.wa.gov. $\mathrm{au}$

For author footnote see end of the article.

Received 7 December 2009

Accepted 23 July 2010

Published Online First

1 October 2010

\section{ABSTRACT \\ Background Early detection of Pseudomonas aeruginosa is essential for successful eradication. The accuracy of serum antibodies against specific and multiple $P$ aeruginosa antigens at predicting lower airway infection in young children with cystic fibrosis (CF) was investigated.}

Methods A commercial $P$ aeruginosa multiple antigen (MAg) ELISA and an in-house exotoxin A (ExoA) ELISA were compared in two populations: a discovery population of 76 children (0.1-7.1 years) undergoing annual bronchoalveolar lavage (BAL)-based microbiological surveillance and a test population of 55 children (0.1-5.6 years) participating in the Australasian CF Bronchoalveolar Lavage Trial.

Results In the discovery population, $P$ aeruginosa was cultured from BAL fluid ( $\geq 10^{5}$ colony-forming units (cfu)/ $\mathrm{ml}$ ) in 15/76 (19.7\%) children (median age 1.88 years). Positive MAg and ExoA serological results were found in $38(50.0 \%)$ and $30(39.5 \%)$ children, respectively. Positive (PPV) and negative (NPV) predictive values for serology at diagnosing $P$ aeruginosa infection $\left(\geq 10^{5} \mathrm{cfu} /\right.$ $\mathrm{ml}$ ) were 0.14 and 0.99 respectively (MAg assay) and 0.11 and 0.98 (ExoA assay). In the test population, $P$ aeruginosa was cultured from BAL fluid $\left(\geq 10^{5} \mathrm{cfu} / \mathrm{ml}\right.$ ) in 16/55 (29.1\%) children (median age 1.86 years) and from oropharyngeal swabs in 32/36 (88.9\%). Positive MAg and ExoA serology was detected in 19 (34.5\%) and $33(60.0 \%)$ children, respectively. The PPV and NPV of serology were 0.26 and 0.94 respectively (MAg assay) and 0.19 and 0.98 (ExoA assay) and were marginally higher for oropharyngeal cultures.

Conclusions Measuring serum antibody responses against $P$ aeruginosa is of limited value for detecting early $P$ aeruginosa infection in young children with $\mathrm{CF}$.

\section{INTRODUCTION}

Lower airway infection with Pseudomonas aeruginosa is an important event in the development of cystic fibrosis (CF) lung disease. However, the early stages of $P$ aeruginosa infection in young children also provides a window of opportunity where aggressive antibiotic treatment may eradicate the organism, delaying its almost inevitable establishment within the lungs and the associated adverse impact upon respiratory function and clinical status. ${ }^{1-10}$ Consequently, there is a strong incentive for early detection of $P$ aeruginosa infection in young children. However, microbiological surveillance in preschool children and infants is difficult owing to their inability to expectorate sputum, and many centres rely upon upper airway cultures which have poor predictive accuracy for lower airway pathogens. ${ }^{11} 12$ As a result, there is renewed interest in serology as a potential diagnostic aid for detecting early lower airway infection with $P$ aeruginosa. ${ }^{13-19}$ The clinical value of serology is likely to be greatest in children too young to expectorate sputum; however, robust data on diagnostic accuracy in this age group are lacking. ${ }^{13-20}$

Bronchoalveolar lavage (BAL) culture is currently accepted as the 'gold standard' technique for detecting lower airway pathogens in young children. ${ }^{21}$ The clinical utility and accuracy of serology in diagnosing $P$ aeruginosa lower airway infection has not been determined in infants and young children with CF using BAL cultures to confirm the presence of lower airway infection. Accordingly, previous reports of serological assays to identify $P$ aeruginosa infection in non-expectorating infants and young children may be unreliable. ${ }^{13-19}$

The optimal panel of antigens for testing remains uncertain and may influence the diagnostic value of serology in young children. Most published data to date report serological responses in older children and adults with CF to a limited panel of $P$ aeruginosa antigens ${ }^{13-15} 1819$ selected to reflect the hierarchical expression of virulence factors and proteins associated with early $P$ aeruginosa tissue invasion. ${ }^{22}$ Studies specifically examining serological responses of infants and preschool children to $P$ aeruginosa antigens are few and have used a limited number of antigens. $^{16} 17$

The present study was performed to test the hypothesis that serum antibodies raised against $P$ aeruginosa antigens would aid the diagnosis of lower airway infection with this pathogen in infants and young children. Specifically, children would seroconvert at the time of lower airway infection with $P$ aeruginos $a$ and measuring serum antibody levels should aid microbiological surveillance. The study compared serological responses using two ELISAs: an in-house $P$ aeruginosa exotoxin A (ExoA) ELISA and a commercially available ELISA containing a blend of 64 different Pseudomonas antigens. We report the diagnostic value of $P$ aeruginosa serology in two independent populations of infants and preschool children with CF: (1) an initial discovery population taking part in the Australian Respiratory Early Surveillance Team for 
Cystic Fibrosis (AREST CF) early surveillance programme (www.ARESTCF.org) that includes annual BAL from diagnosis as part of routine clinical microbiological surveillance; and (2) a test population recruited to the Australasian Cystic Fibrosis Bronchoalveolar Lavage (ACFBAL) trial (http://www.actr.org. $\mathrm{au} /$ ) who underwent BAL sampling at recruitment and to direct treatment during lower airway exacerbations.

\section{METHODS}

Full details are given in the online supplement.

\section{Study subjects}

The discovery population comprised all children with CF aged $<7$ years in Western Australia enrolled in a BAL-based early surveillance programme. BAL fluid and serum samples were obtained soon after diagnosis and annually until 7 years of age. Approval was given by the Human Ethics Committee of Princess Margaret Hospital for Children and parents gave written consent for each bronchoscopy.

The test population comprised children recruited to the ACFBAL study. Children randomised to BAL-directed therapy underwent routine BAL, oropharyngeal (OP) swab and serum sampling before 6 months of age and during infective exacerbations. Children in both trial arms underwent BAL at 5 years of age and OP swabs and serum were collected at that time. Approval for the ACFBAL study was obtained from the ethics committees of the University of Queensland and from participating centres.

\section{Bronchoscopy and BAL fluid sampling}

Flexible bronchoscopy was performed under general anaesthesia. Aliquots of $1 \mathrm{ml} / \mathrm{kg}$ non-bacteriostatic normal saline were instilled with the bronchoscope wedged into the right middle lobe (RML) bronchus. In the discovery population three aliquots were used and the first was sent to the microbiology laboratory. In the test population a single aliquot was also obtained from the lingula and pooled with that from the RML.

\section{Microbiology}

BAL fluid was cultured using standard microbiological techniques. Children with BAL cultures that grew $P$ aeruginosa at a bacterial density $\geq 10^{5}$ colony-forming units $(\mathrm{cfu}) / \mathrm{ml}$ were considered to be infected. ${ }^{11}$ Definitions of chronic and intermittent infection with $P$ aeruginos $a$ were adapted from the Leeds criteria: $P$ aeruginosa infection was categorised as intermittent where $\leq 50 \%$ of all BAL fluid cultures were positive and as persistent/chronic where $>50 \%$ were positive. ${ }^{23}$ This classification was also used to categorise intermittent and chronic colonisation in OP cultures in this study. A secondary analysis was undertaken including Paeruginosa of any density cultured in BAL fluid.

\section{Serology}

Serum antibodies directed against $P$ aeruginosa were measured using two assays: (1) a commercially available semiquantitative IgG ELISA consisting of a multiple antigenic (MAg) blend of 64 different Pseudomonas antigens from 17 of the most common $P$ aeruginosa serotypes (Statens Serum Institut, Denmark) and (2) an optimised and validated in-house $P$ aeruginosa ExoA IgG-ELISA (see online supplement for details).

\section{Determining diagnostic accuracy}

Positive control serum was used to optimise the ELISA assays. The ability of serology to detect infection with $P$ aeruginosa was assessed using receiver operator characteristic (ROC) curves. In both assays serum anti-Pseudomonas antibody levels from children with CF (aged 4-15 years) with established persistent $P$ aeruginosa lower airway infection were compared with levels from the negative control serum. The optimal discrimination between positive and negative controls was obtained at a 1:256 dilution of serum and determined by calculating sensitivity and specificity for differing threshold values and choosing the titre producing the best balance between sensitivity and specificity and the largest area under the ROC curve. The optimal threshold antibody level indicating a positive test for the $\mathrm{MAg}$ assay was 3.1 arbitrary units (AU), and 1.7 AU for the ExoA assay. The area under the ROC curve was 0.98 (95\% CI 0.94 to 1.0) for the MAg assay and 0.98 (95\% CI 0.95 to 1.0$)$ for the ExoA assay (figure 1). The intra- and interassay coefficients of variability were acceptable and confirmed as $1.97 \%$ and $0.33 \%$, respectively, for the $\mathrm{MAg}$ assay and $1.25 \%$ and $8.0 \%$ for the ExoA assay. These optimal (cut-off) values were then used to categorise children in the discovery population as being 'positive' or 'negative' for $P$ aeruginosa antibodies. The sensitivity, specificity and positive (PPV) and negative (NPV) predictive values of serology as markers of $P$ aeruginosa infection were determined from contingency tables and $95 \%$ CIs calculated using the binomial exact method. The process was repeated in the test population.
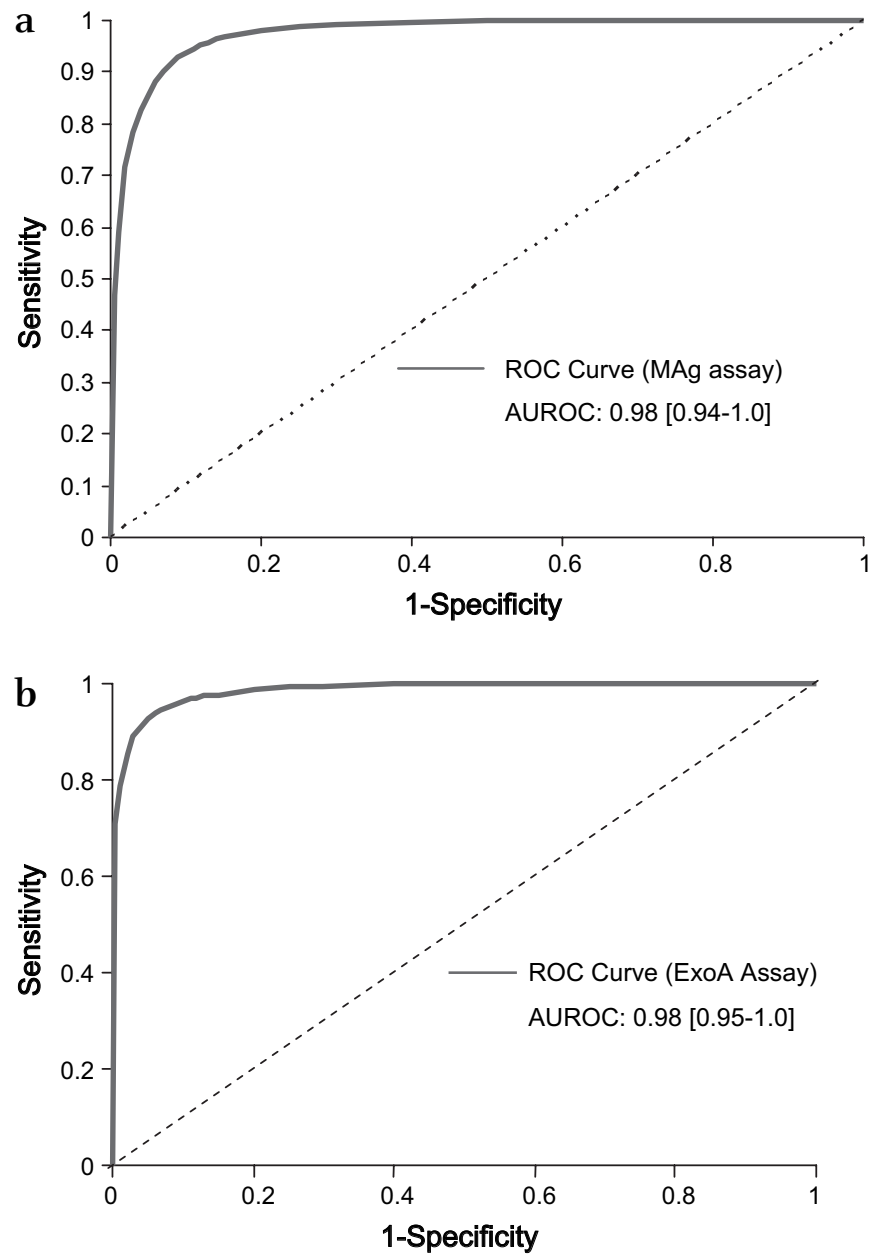

Figure 1 Receiver operator characteristic (ROC) curves for antiPseudomonas serological tests distinguishing between the presence or absence of Pseudomonas aeruginosa in culture-confirmed chronically infected patients with cystic fibrosis and healthy controls using 1:256 dilution of serum. AUROC, area under the ROC curve. 


\section{Statistical analysis}

Correlations between independent variables were performed using clustered robust SE to adjust for potential intraclass correlations between observations within the dataset. Variables were log-transformed to achieve normality and results expressed as regression coefficients (SE) and 95\% CIs. Probability values $<0.05$ were considered statistically significant. Statistical analyses were completed using Stata software Version 10.

\section{RESULTS \\ Discovery population}

One hundred and eighty-six paired serum and BAL fluid cultures from 76 children aged $0.1-7.1$ years were analysed (table 1). $P$ aeruginosa was cultured from BAL fluid at any bacterial density in 21 children $(27.6 \%$ ), median age 2.2 years (IOR 1.3-3.3). Mucoid $P$ aeruginosa was cultured in seven BAL fluid samples from six children. Fifteen children (19.7\%), median age 1.88 years (IOR $1.05-4.30)$ were infected $\left(\geq 10^{5} \mathrm{cfu} / \mathrm{ml}\right)$ with $P$ aeruginosa. One child was infected persistently with $P$ aeruginosa and the remaining 14 subjects were infected intermittently (table 1). Only 10 of the 21 children (47.6\%) had respiratory symptoms (predominantly moist cough) at the time Paeruginosa (at any bacterial density) was detected. All patients with positive BAL cultures for $P$ aeruginosa underwent treatment for eradication of Pseudomonas (see online supplement for details). No child received regular or cycled inhaled tobramycin treatment during the study.

The MAg and ExoA assays provided positive serological results in $38(50.0 \%)$ and $30(39.5 \%)$ children in the discovery population, respectively. In those subjects with serial assessments of serology available from CF diagnosis following newborn screening $(n=14)$, the median ages for the first positive test results were 1.30 and 1.20 years by Mag and ExoA assays, respectively. There was a wide range in individual antibody titres (0.40-94.6 AU) and titres correlated with increasing age regardless of lower airway infection with $P$ aeruginosa (MAg: coefficient (SE) 1.84 (0.29); 95\% CI 1.26 to 2.42; p<0.001), ExoA: coefficient (SE) 1.10 (0.29); $95 \%$ CI 0.51 to $1.69 ; \mathrm{p}<0.001)$. There was no correlation between bacterial density from BAL cultures and the antibody titre MAg (coefficient (SE) -0.266 ( \pm 0.846 ), $95 \% \mathrm{CI}-1.92$ to $1.39, \mathrm{p}=0.753$ ) and ExoA (coefficient (SE) -0.227 ( \pm 0.648 ), $95 \% \mathrm{CI}-1.50$ to $1.04, \mathrm{p}=0.726$ ) (see figure 1 in the online supplement). Regardless of their subsequent infection status, following seroconversion most children in the discovery population who provided multiple serum samples had persistent
$P$ aeruginosa antibodies detected by both ExoA and MAg assays (table 1).

The sensitivity, specificity and predictive values of serology for lower airway infection with $P$ aeruginosa in the discovery population are shown in table 2. The PPVs were poor, being 0.14 (95\% CI 0.06 to 0.23 ) for MAg and 0.11 (95\% CI 0.04 to 0.18 ) for Exo A. The NPVs for both assays were high, being 0.99 (95\% CI 0.97 to 1.0$)$ and 0.98 (95\% CI 0.95 to 1.0$)$, respectively. These data were recalculated incorporating any growth of $P$ aeruginosa in BAL fluid cultures. As shown in table 3, the PPV improved only marginally while the NPV remained high.

\section{Test population}

One hundred and sixty-two paired serum and BAL fluid samples were obtained prospectively from 55 children (age $0.1-5.6$ years) randomised to the BAL-directed treatment arm of the ACFBAL trial. The median age at first BAL sampling was 0.39 years (table 1) and subjects underwent a median of 5 (range 1-13) BAL procedures during the study period. OP sampling during an infective exacerbation in the ACFBAL study was occasionally missed by some participating centres and therefore these data are incomplete. A total of 89 OP swabs were obtained at the same time as the BAL fluid from 36 children, median age 2.47 years (range $0.25-5.27$ ).

$P$ aeruginosa at any density was isolated in 68 BAL fluid samples from 31 children (56.4\%). In 29 children (93.5\%) $P$ aeruginosa was detected only intermittently. Sixteen $(29.1 \%)$ grew $\geq 10^{5} \mathrm{cfu} / \mathrm{ml} P$ aeruginosa in their BAL fluid at a median age of 1.86 years (table 1). Bacterial counts of $P$ aeruginosa were generally low at their initial detection: $10^{2}-10^{4} \mathrm{cfu} / \mathrm{ml}$ in 15 subjects (48.4\%), $10^{5}-10^{6} \mathrm{cfu} / \mathrm{ml}$ in 10 subjects $(32.3 \%)$ and $\geq 10^{7} \mathrm{cfu} / \mathrm{ml}$ in 6 subjects $(19.3 \%)$. Mucoid $P$ aeruginosa strains were isolated in $6 / 68(8.8 \%)$ BAL cultures from 5 children. Of the 36 subjects who provided OP swabs, 32 (88.9\%) cultured $P$ aeruginosa from $\mathrm{OP}$ swabs at a median age of first detection of 2.0 years (IOR $0.2-4.9)$. Almost all of these 32 children $(n=30$, $94 \%$ ) were intermittently colonised with $P$ aeruginosa ( $\leq 50 \%$ of all OP cultures positive for $P$ aeruginosa since first isolation) in their upper airways. Only two children were chronically colonised ( $>50 \%$ of all OP cultures since first isolation) with $P$ aeruginosa in their upper airways. The concordance between positive cultures of $P$ aeruginosa in the upper airways and simultaneous isolation in BAL fluid was poor in the test population overall. A positive culture for $P$ aeruginosa in the upper airway was associated with concomitant culture of $P$ aeruginosa in the BAL fluid in $53 \%$ of paired samples. The predictive values

Table 1 Demographic and clinical characteristics of the discovery and test populations

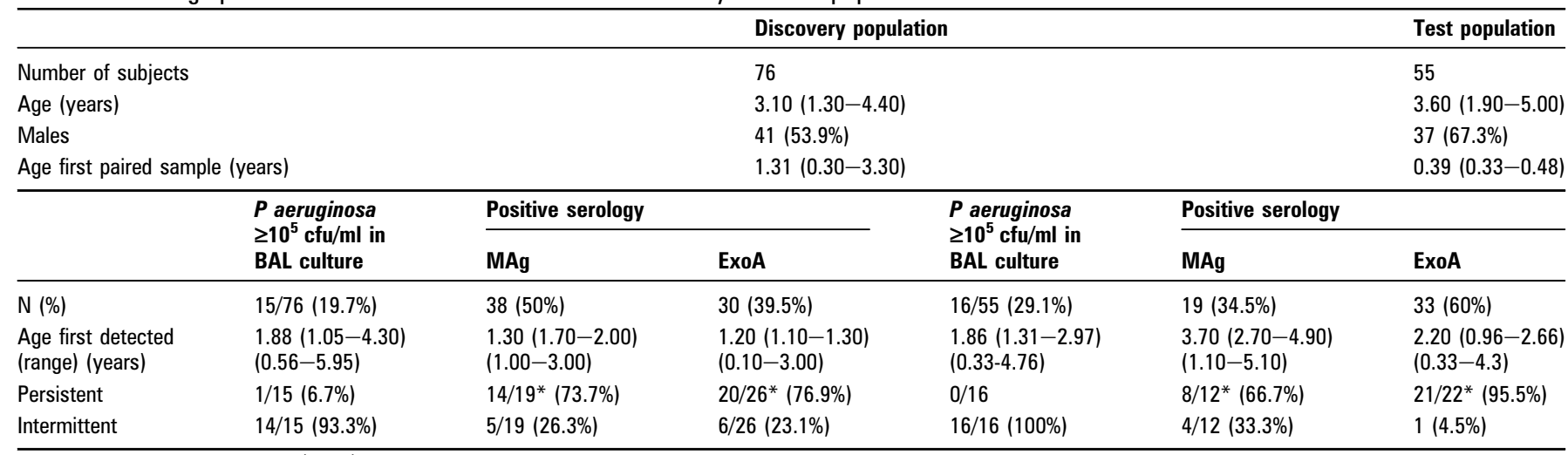

Results presented as median $\left(25^{\text {th }}-75^{\text {th }}\right.$ centile) unless otherwise specified.

*Subjects with $>2$ consecutive serology samples.

BAL, bronchoalveolar lavage; $\mathrm{MAg}$, multiple blended $P$ aeruginosa antigens; ExoA, $P$ aeruginosa exotoxin $A$ antigen. 
Table 2 Positive (PPV) and negative (NPV) predictive values (95\% Cls) for serology as an indicator of $P$ aeruginosa $\geq 10^{5}$ colony-forming units/ml in bronchoalveolar lavage (BAL) fluid cultures

\begin{tabular}{|c|c|c|c|c|c|}
\hline & $\mathbf{N}$ & Sensitivity & Specificity & PPV & NPV \\
\hline \multicolumn{6}{|c|}{ Multiple $P$ aeruginosa antigens } \\
\hline \multicolumn{6}{|l|}{ BAL } \\
\hline Discovery population & 76 & 0.91 (0.74 to 1.0$)$ & $0.64(0.57$ to 0.72$)$ & $0.14(0.06$ to 0.23$)$ & $0.99(0.97$ to 1.0$)$ \\
\hline Test population & 56 & $0.53(0.28$ to 0.79$)$ & $0.82(0.75$ to 0.88$)$ & $0.26(0.1$ to 0.41$)$ & 0.94 (0.89 to 0.98$)$ \\
\hline \multicolumn{6}{|l|}{$P$ aeruginosa exotoxin $\mathrm{A}$} \\
\hline \multicolumn{6}{|l|}{ BAL } \\
\hline Discovery population & 76 & $0.82(0.59$ to 1.0$)$ & $0.57(0.485$ to 0.65$)$ & $0.11(0.04$ to 0.18$)$ & $0.98(0.95$ to 1.0$)$ \\
\hline Test population & 56 & $0.93(0.80$ to 1.0$)$ & $0.52(0.43$ to 0.61$)$ & $0.19(0.10$ to 0.28$)$ & 0.98 (0.96 to 1.0$)$ \\
\hline
\end{tabular}

of OP cultures for $P$ aeruginosa in BAL fluid cultures were correspondingly poor: PPV $0.54(0.4-0.67)$ and NPV 0.84 (0.77-0.91). As in the discovery population, patients with positive BAL cultures for $P$ aeruginosa underwent treatment for eradication of Pseudomonas (for details see online supplement), and no child received regular or cycled inhaled tobramycin treatment during the study.

In this population of patients with CF, positive MAg ELISA serology results were present in $24 / 55$ subjects (43.6\%) and in $46 / 162$ serum samples (28.4\%). In contrast, 33 subjects $(60 \%)$ had positive serology to ExoA antigens. Following an initial seroconversion, most subjects who provided repeated serum samples remained seropositive. No correlation was observed between $P$ aeruginosa colony counts in BAL fluid and antibody titre (ExoA, $\mathrm{r}^{2}=0.03, \mathrm{p}=0.12 ; \mathrm{MAg}, \mathrm{r}^{2}=0.02, \mathrm{p}=0.14$ ).

The sensitivity, specificity and predictive accuracy of serology at diagnosing $P$ aeruginosa lower airway infection are shown in table 2 and for any growth of $P$ aeruginosa in BAL fluid in table 3. PPVs were universally poor with both assays (range $0.10-0.41$ ) and were worse at higher bacterial density. The NPVs produced by both assays were high (range 0.94-0.99) at bacterial densities $\geq 10^{5} \mathrm{cfu} / \mathrm{ml}$.

Overall, positive serology was marginally better at predicting $P$ aeruginosa in OP than BAL cultures (table 3 ). The PPV of the MAg assay (0.57) was slightly higher than that obtained from the in-house ExoA assay (0.43). The NPV for serology from the MAg assay was lower for OP than BAL cultured specimens. The diagnostic value of serology for positively predicting Paeruginosa in either BAL or OP cultures did not vary significantly with age.

\section{DISCUSSION}

This is the first study to examine the diagnostic value of serology as a less invasive marker of lower airway infection with $P$ aeruginosa in two independent populations of infants and preschool children with CF using BAL culture as the microbiological reference standard. By employing BAL to detect respiratory infection, we have attempted to address uncertainties regarding the validity of serology testing in young children. In this current study the PPV of serology using both single and multiple antigen ELISA assays was too poor to usefully serve as a less invasive marker of lower airway infection with $P$ aeruginosa in young children with CF. Neither in the population of clinically stable predominantly asymptomatic infants and preschool children undergoing routine annual BAL surveillance nor in acutely symptomatic young children experiencing a respiratory exacerbation was the diagnostic value of serology sufficiently reliable to diagnose $P$ aeruginosa infection with any certainty. The prevalence of culture-proven $P$ aeruginosa infection in the two populations was consistent with previously reported ranges in preschool children. ${ }^{124} 25$ The high NPVs observed for both assays in the discovery cohort suggest that serology may have clinical value in excluding lower airway infection with $P$ aeruginosa in young children undergoing routine pathogen surveillance. However, in a population with a higher prevalence of $P$ aeruginosa infection, the NPV of serology using either assay is likely to be too low to reliably exclude the presence of lower airway infection in acutely symptomatic children. These children will therefore still require BAL culture to determine whether they are infected with $P$ aeruginos $a$ and whether specific antipseudomonal treatment is needed. In addition, the association

Table 3 Positive (PPV) and negative (NPV) predictive values (95\% Cls) for serology as an indicator of $P$ aeruginosa at any density in bronchoalveolar lavage (BAL) fluid cultures and its presence in oropharyngeal (OP) cultures

\begin{tabular}{llllll}
\hline & N & Sensitivity & Specificity & PPV & NPV \\
\hline $\begin{array}{l}\text { Multiple } P \text { aeruginosa antigens } \\
\text { BAL }\end{array}$ & & & & & \\
$\quad \begin{array}{l}\text { Discovery population } \\
\quad\end{array}$ & 76 & $0.95(0.85$ to 1.0$)$ & $0.62(0.55$ to 0.69$)$ & $0.23(0.14$ to 0.32$)$ & $0.99(0.97$ to 1.0$)$ \\
$\quad \begin{array}{l}\text { Test population } \\
\text { OP }\end{array}$ & 56 & $0.46(0.3$ to 0.62$)$ & $0.82(0.75$ to 0.88$)$ & $0.43(0.27$ to 0.58$)$ & $0.84(0.77$ to 0.90$)$ \\
$\quad$ Test population & 32 & $0.32(0.14$ to 0.50$)$ & $0.88(0.79$ to 0.96$)$ & $0.57(0.36$ to 0.78$)$ & $0.76(0.67$ to 0.86$)$ \\
$\begin{array}{l}\text { P aeruginosa exotoxin A } \\
\text { BAL }\end{array}$ & & & & & \\
$\quad \begin{array}{l}\text { Discovery population } \\
\text { Test population }\end{array}$ & 76 & $0.90(0.77$ to 1.0$)$ & $0.57(0.5$ to 0.64$)$ & $0.20(0.12$ to 0.28$)$ & $0.98(0.95$ to 1.0$)$ \\
OP & 55 & $0.84(0.72$ to 0.96$)$ & $0.52(0.36$ to 0.61$)$ & $0.34(0.24$ to 0.44$)$ & $0.92(0.85$ to 0.98$)$ \\
$\quad$ Test population & 32 & $0.88(0.75$ to 1.0$)$ & $0.55(0.42$ to 0.67$)$ & $0.43(0.30$ to 0.57$)$ & $0.92(0.84$ to 1.0$)$ \\
\hline
\end{tabular}


of positive serological tests with upper airway colonisation means that a positive result does not always indicate that lower airway infection is present.

In the present study we considered $\geq 10^{5} \mathrm{cfu} / \mathrm{ml} P$ aeruginosa in BAL fluid as indicating infection. ${ }^{11}$ However, many physicians consider the presence of any $P$ aeruginosa in the lungs sufficient indication to instigate eradication therapy. We therefore recalculated the PPV and NPV of serology using both assays. While the PPV increased marginally in both the test and discovery populations and the NPV decreased somewhat, the accuracy of serology remains too low to be of use clinically. Moreover, the predictive accuracy of serology at detecting upper airway colonisation was greater than for lower airway infection. These data show that serology does not improve upon the predictive value of positive OP swabs as indicators of lower airway infection with $P$ aeruginosa.

There is no international consensus regarding the most appropriate definition of persistent and intermittent infection with $P$ aeruginosa detected in upper airway cultures. Chronic and intermittent infection is even less well-defined in populations of young children where $P$ aeruginosa is detected using BAL culture during annual pulmonary surveillance or infective exacerbations. We based the definition of persistent and intermittent infection in BAL culture for this current study on the Leeds criteria ${ }^{15}{ }^{23}$ for reasons of simplicity, although it must be acknowledged that the clinical implications of chronic infection as defined in this current study may not be equivalent to those observed in studies using upper airway cultures to detect $P$ aeruginosa.

The optimal antigen panel for detecting early $P$ aeruginosa infection remains uncertain. ExoA was chosen as the single antigen ELISA in this study as antibodies to ExoA are among the first to be detected in patients with CF with $P$ aeruginosa infection ${ }^{17} 22$ and because of the reported association between elevated titres to ExoA and clinical status. ${ }^{26}$ To date, many investigators have measured responses to a small range of individual antigens (ExoA, elastase, alkaline phosphatase, flagellin, exoenzyme $S$, outer membrane proteins) and some also report enhanced sensitivity of serology when individual responses to single $P$ aeruginos $a$ antigens are considered in combination rather than independently. ${ }^{13-15} 27-29$ In the current study we examined whether measurement of responses to a broad range of antigens in combination would enhance the diagnostic value of serology at detecting lower airway infection with $P$ aeruginosa in young children compared with a single antigen assay. Clearly this was not the case, and the predictive values of either assay were insufficient to avoid young children undergoing BAL for collection of lower airway secretions for culture. Further studies are needed to identify and to validate the most suitable antigen panels for screening preschool children before serology can be recommended as a reliable diagnostic tool in the detection of early $P$ aeruginosa CF lung infection. ${ }^{30}$

Previous studies report much higher PPVs and NPVs of serology within heterogeneous populations of $\mathrm{CF}^{13-15} 27$ The higher prevalence of $P$ aeruginosa infection and the inclusion of predominantly older chronically infected patients may account for the higher predictive values in these studies. Antibody levels in chronically infected older patients are almost universally elevated, with titres increasing as lung disease severity progresses. $^{27} 28$ Cut-off titres that distinguish positive from negative $P$ aeruginosa infection calculated from adult-based serology pools are likely to be too insensitive to apply to a young paediatric population with early infection from non-mucoid strains, relatively mild lung disease and low immunological responses to the initial infecting strains.
While microbiological culture of BAL is considered the present reference standard for detecting lower airway pathogens, it is not necessarily the 'ideal' standard or without limitations. Bronchoscopy and BAL is a relatively invasive procedure requiring general anaesthesia and day case admission, which limits the frequency with which it may be used in lower airway pathogen surveillance in children. We acknowledge that a negative culture does not provide unequivocal assurance that $P$ aeruginosa infection is not present in the lung due to the limitations of regional sampling and reliance on viable bacteria in secretions being accessible to bronchoscopic sampling. ${ }^{31} \mathrm{We}$ recognise the limitations of using BAL cultures as a gold standard given their potential failure to identify pathogens at low densities and if the patient is receiving antibiotic treatment. Highly sensitive molecular-based techniques such as quantitative PCR (qPCR) assays show considerable promise for overcoming these difficulties. ${ }^{32} 33$ However, the techniques and expertise to employ qPCR as a means of improving bacterial detection are not widely available to CF clinicians and are yet to be validated in populations of young children undergoing BAL.

BAL samples may also not always provide accurate information regarding quantitative bacterial counts in the lungs. Intraindividual variability in quantitative bacterial counts has been described in adults undergoing sputum and BAL sampling, ${ }^{34}$ and it is possible that any significant relationship between $P$ aeruginosa density and the magnitude of serological titres may be obscured in this present study. An equally likely explanation for the poor association between bacterial density and serological titre, we believe, is that antibody levels are dependent on individual variations in humoral host responses to invasive pathogens rather than bacterial load alone. Finally, contamination of BAL samples by upper airway secretions is still possible, although every attempt was made to avoid this by using laryngeal masks and not suctioning respiratory secretions until the tip of the bronchoscope was below the carina and, if possible, wedged into the target bronchus.

We are unable to comment on the prognostic value of increasing serology titres in the months before the initial and subsequent detection of $P$ aeruginosa in BAL cultures in either population as serology and BAL sampling were performed only annually (biannually at most) in the discovery population and were predominantly symptom-driven in the test population. The potential for frequent serological testing in combination with regular OP swab collection for culture or molecular-based detection methods as a means of diagnosing lower airway infection with $P$ aeruginosa requires further investigation in larger populations. ${ }^{32}$ However, both serial OP and blood sampling are not always well tolerated by all young children. Furthermore, any such study still needs BAL as the 'gold standard' for confirmation of a lower airway infection.

In conclusion, the data from the present study argue against using $P$ aeruginosa serological testing as a substitute for lower airway culture in either the setting of pathogen surveillance or respiratory exacerbation among infants and preschool children with CF.

\section{Author footnote}

Members of the Australian Respiratory Early Surveillance Team for Cystic Fibrosis (AREST CF): E Balding, L Berry, S Brennan, C Calogero, R Carzino, N De Klerk,

T A Douglas, C Gangell, L Garratt, G Hall, A Kicic, I Laing, B Linnane, J Massie, L Mott, D Mullane, C Murray, G Nolan, N Pillarisetti, S Ranganathan, C Robertson, P Robinson, B Skoric, P D Sly, S M Stick, E Sutanto. Members of the Australasian Cystic Fibrosis Bronchoalveolar Lavage (ACFBAL) study: D S Armstrong, C A Byrnes, J B Carlin, D M Cooper, P J Cooper, C J Dakin, P W Francis, K Grimwood, A J Martin,

R J Massie, I B Masters, L K Patterson, S Ranganathan, C F Robertson, I F Robertson, P J Robinson, S Vidmar, C E Wainwright, B F Whitehead. 
Acknowledgements The authors acknowledge the valuable contribution of Charles Goh who performed the initial optimisation of the ELISAs and Catherine Gangell for her support in the data presentation.

Funding Australian Cystic Fibrosis Research Trust and National Health and Medical Research Council of Australia.

Competing interests None.

Ethics approval This study was conducted with the approval of the Princess Margaret Hospital for Children ethics committee.

Provenance and peer review Not commissioned; externally peer reviewed.

\section{REFERENCES}

1. Anon. Cystic Fibrosis Foundation Patient Registry 2007 Annual Data Report. Bethesda, Maryland, 2008.

2. Frederiksen B, Koch C, Hoiby N. Antibiotic treatment of initial colonization with Pseudomonas aeruginosa postpones chronic infection and prevents deterioration of lower airway function in cystic fibrosis. Pediatr Pulmonol 1997;23:330-5.

3. Gibson RL, Emerson J, Mayer-Hamblett N, et al. Duration of treatment effect after tobramycin solution for inhalation in young children with cystic fibrosis. Pediatr Pulmonol 2007;42:610-23.

4. Griese M, Muller I, Reinhardt D. Eradication of initial Pseudomonas aeruginosa colonization in patients with cystic fibrosis. Eur J Med Res 2002:7:79-80.

5. Hudson VL, Wielinski CL, Regelmann WE. Prognostic implications of initial oropharyngeal bacterial flora in patients with cystic fibrosis diagnosed before the age of two years. J Pediatr 1993;122:854-60.

6. Kosorok MR, Zeng L, West SE, et al. Acceleration of lung disease in children with cystic fibrosis after Pseudomonas aeruginosa acquisition. Pediatr Pulmonol 2001:32:277-87.

7. Li Z, Kosorok MR, Farrell PM, et al. Longitudinal development of mucoid Pseudomonas aeruginosa infection and lung disease progression in children with cystic fibrosis. JAMA 2005;293:581-8.

8. Nixon GM, Armstrong DS, Carzino $R$, et al. Clinical outcome after early Pseudomonas aeruginosa infection in cystic fibrosis. J Pediatr 2001;138:699-704.

9. Taccetti G, Campana S, Festini F, et al. Early eradication therapy against Pseudomonas aeruginosa in cystic fibrosis patients. Eur Respir J 2005:26:458-61.

10. Emerson J, Rosenfeld M, McNamara S, et al. Pseudomonas aeruginosa and other predictors of mortality and morbidity in young children with cystic fibrosis. Pediatr Pulmonol 2002:34:91-100.

11. Armstrong DS, Grimwood K, Carlin JB, et al. Bronchoalveolar lavage or oropharyngeal cultures to identify lower airway pathogens in infants with cystic fibrosis. Pediatr Pulmonol 1996:21:267-75.

12. Rosenfeld M, Emerson J, Accurso F, et al. Diagnostic accuracy of oropharyngeal cultures in infants and young children with cystic fibrosis. Pediatr Pulmonol 1999:28:321-8.

13. Kappler M, Kraxner A, Reinhardt D, et al. Diagnostic and prognostic value of serum antibodies against Pseudomonas aeruginosa in cystic fibrosis. Thorax 2006;61:684-8.

14. Ratjen F, Walter $\mathrm{H}$, Haug $\mathrm{M}$, et al. Diagnostic value of serum antibodies in early Pseudomonas aeruginosa infection in cystic fibrosis patients. Pediatr Pulmonol 2007:42:249-55.
15. Tramper-Stranders GA, van der Ent CK, Slieker MG, et al. Diagnostic value of serological tests against Pseudomonas aeruginosa in a large cystic fibrosis population. Thorax 2006;61:689-93.

16. Burns JL, Gibson RL, McNamara S, et al. Longitudinal assessment of Pseudomonas aeruginosa in young children with cystic fibrosis. J Infect Dis 2001;183:444-52

17. West SE, Zeng L, Lee BL, et al. Respiratory infections with Pseudomonas aeruginosa in children with cystic fibrosis: early detection by serology and assessment of risk factors. JAMA 2002;287:2958-67.

18. Pressler T, Karpati $\mathrm{F}$, Granstrom M, et al. Diagnostic significance of measurements of specific IgG antibodies to Pseudomonas aeruginosa by three different serological methods. J Cyst Fibros 2009:8:37-42.

19. Rao AR, Laxova A, Farrell PM, et al. Proteomic identification of OprL as a seromarke for initial diagnosis of Pseudomonas aeruginosa infection of patients with cystic fibrosis. J Clin Microbiol 2009;47:2483-8.

20. Farrell PM, Govan JR. Pseudomonas serology: confusion, controversy, and challenges. Thorax 2006;61:645-7.

21. Brennan S, Gangell C, Wainwright $C$, et al. Disease surveillance using bronchoalveolar lavage. Paediatr Respir Rev 2008;9:151-9.

22. Doring G, Hoiby N. Longitudinal study of immune response to Pseudomonas aeruginosa antigens in cystic fibrosis. Infect Immun 1983:42:197-201.

23. Lee TW, Brownlee KG, Denton M, et al. Reduction in prevalence of chronic Pseudomonas aeruginosa infection at a regional pediatric cystic fibrosis center. Pediatr Pulmonol 2004:37:104-10.

24. UK Cystic Fibrosis Trust Database. Annual Data Report. UK CF Database 2006 Scotland: University of Dundee, 2004.

25. Cystic Fibrosis Australia. Cystic Fibrosis in Australia 2007. 10th Annual Report from the Australasian Cystic Fibrosis Registry. North Ryde, Australia: Cystic Fibrosis Australia, 2009

26. Jagger K, Robinson D, Franz $M$, et al. Detection by enzyme-linked immunosorbent assays of antibody specific for Pseudomonas protease and exotoxin A in sera from cystic fibrosis patients. J Clin Microbiol 1982:15:1054-8.

27. Pedersen SS, Espersen F, Hoiby N. Diagnosis of chronic Pseudomonas aeruginosa infection in cystic fibrosis by enzyme-linked immunosorbent assay. J Clin Microbiol 1987:25:1830-6.

28. Brett MM, Ghoneim AT, Littlewood JM. Serum antibodies to Pseudomonas aeruginosa in cystic fibrosis. Arch Dis Child 1986;61:1114-20.

29. Brett MM, Ghoneim AT, Littlewood JM. Serum IgG antibodies in patients with cystic fibrosis with early Pseudomonas aeruginosa infection. Arch Dis Child 1987;62:357-61.

30. Montor WR, Huang J, Hu Y, et al. Genome-wide study of Pseudomonas aeruginosa outer membrane protein immunogenicity using self-assembling protein microarrays. Infect Immun 2009:77:4877-86.

31. Gutierrez JP, Grimwood K, Armstrong DS, et al. Interlobar differences in bronchoalveolar lavage fluid from children with cystic fibrosis. Eur Respir $\mathrm{J}$ 2001:17:281-6.

32. da Silva Filho L, Tateno A, Martins K, et al. The combination of PCR and serolog increases the diagnosis of Pseudomonas aeruginosa colonisation/infection in cystic fibrosis. Pediatr Pulmonol 2007:42:938-44.

33. Deschaght $\mathbf{P}$, De Baere T, Van Simaey L, et al. Comparison of the sensitivity of culture, PCR and quantitative real-time PCR for the detection of Pseudomonas aeruginosa in sputum of cystic fibrosis patients. BMC Microbiol 2009;9:244

34. Fujitani S, Yu VL. Quantitative cultures for diagnosing ventilator-associated pneumonia: a critique. Clin Infect Dis 2006:43(Suppl 2):S106-13. 